\title{
3D Printed Nervous System on a Chip
}

\author{
Blake N. Johnson ${ }^{a, b}$, Karen Z. Lancaster ${ }^{c}$, lan B. Hogue ${ }^{c}$, Fanben Meng ${ }^{b, d}$, Yong Lin Kong ${ }^{b}$, \\ Lynn W. Enquist ${ }^{\mathrm{c}}$, and Michael C. McAlpine ${ }^{\mathrm{b}, \mathrm{d},{ }^{*}}$ \\ aDepartment of Industrial and Systems Engineering, Virginia Tech, Blacksburg, Virginia 24061, \\ United States \\ ${ }^{b}$ Department of Mechanical and Aerospace Engineering, Princeton University, Princeton, New \\ Jersey 08544, United States \\ 'Department of Molecular Biology and Princeton Neuroscience Institute, Princeton University, \\ Princeton, New Jersey 08544, United States \\ dDepartment of Mechanical Engineering, University of Minnesota, Minneapolis, Minnesota 55455, \\ United States
}

\begin{abstract}
Bioinspired organ-level in vitro platforms are emerging as effective technologies for fundamental research, drug discovery, and personalized healthcare. In particular, models for nervous system research are especially important, due to the complexity of neurological phenomena and challenges associated with developing targeted treatment of neurological disorders. Here we introduce an additive manufacturing-based approach in the form of a bioinspired, customizable 3D printed nervous system on a chip (3DNSC) for the study of viral infection in the nervous system. Micro-extrusion 3D printing strategies enabled the assembly of biomimetic scaffold components (microchannels and compartmented chambers) for the alignment of axonal networks and spatial organization of cellular components. Physiologically relevant studies of nervous system infection using the multiscale biomimetic device demonstrated the functionality of the in vitro platform. We found that Schwann cells participate in axon-to-cell viral spread but appear refractory to infection, exhibiting a multiplicity of infection (MOI) of 1.4 genomes per cell. These results suggest that 3D printing is a valuable approach for the prototyping of a customized model nervous system on a chip technology.
\end{abstract}

\section{Introduction}

The development of hierarchal organ-level platforms, known as organs-on-chips, ${ }^{1-5}$ as potential complements and replacements for the extensive use of small animal models, has arisen as a critical area in fundamental and clinical research. ${ }^{4}$ To date, organs-on-chips have

*mcalpine@umn.edu.

Electronic Supplementary Information (ESI) available: Micrographs of 3DNSC fabrication, viable cell printing, scanning electron micrograph characterization of 3D printed microchannels on protein-coated substrates, rapid prototyping of the 3DNSCs, comparison of viral transport imaging on different substrate materials, and association of Schwann cells and axons within microchannels.

Supporting Information is available online or from the author. 
been primarily developed via microfabrication, which has led to a number of impressive demonstrations. ${ }^{1,3,6-8}$ Yet, this approach requires subsequent biofunctionalization and validation steps due to processing conditions which are not amenable to biological functionalities. It would be desirable to merge the capabilities of computer-driven material assembly, rapid prototyping, and controlled biofunctionalization in a one-pot manufacturing approach for in vitro systems.

3D printing has evolved as an enabling technology for the development of advanced materials and devices, ${ }^{9-13}$ with applications in conformal electronics, ${ }^{14-16}$ biomedical devices, ${ }^{17-19}$ and prosthetics. ${ }^{20,21}$ The scope of 3D printed materials is expanding, and already includes smart materials, ${ }^{22}$ nanomaterials, ${ }^{14,16,21}$ biomaterials, ${ }^{21}$ living materials (e.g. cell-laden matrices), ${ }^{23}$ and combinations of these for novel biotechnologies. For example, tissue constructs, ${ }^{17,24-26}$ organs, ${ }^{2,26,27}$ biosensors, ${ }^{28,29}$ actuators, ${ }^{30}$ and even bionic organs ${ }^{21}$ have recently been fabricated. The palette of fundamental materials research and novel applications in the 3D printing space is rapidly evolving. Indeed, 3D printing may offer a potential solution for developing next-generation organon-a-chip technologies, ${ }^{31}$ as it provides a bottom-up manufacturing paradigm for chip customization, rapid prototyping, and multi-material processing capabilities.

Among the organ systems of interest - including the cardiovascular, digestive, endocrine, muscular, nervous, respiratory, and skeletal systems ${ }^{6,32-34}$ - the development of in vitro models for the nervous system is vital, as the treatment of neurological disorders is a critical medical challenge. As a result, various microfluidic-based platforms have been examined for neuroscience applications. ${ }^{35,36}$ An automated biomanufacturing approach which integrates the fabrication and biofunctionalization steps in an interwoven format could allow for enhanced design customization and improve the accuracy of the resultant model.

Here we describe a multiscale, 3D printed biomimetic system that reconstitutes the critical function of glial cell-axon interfaces in the nervous system. Our bioinspired 3D printed device models various aspects of the complex and integrated organ-level responses to viral infections occurring within the nervous system. The device includes 3D printed topography ${ }^{37}$ in the form of microchannels for axonal alignment and compartmented chambers for cell isolation. Viral infection studies suggest that Schwann cells participate in axon-to-cell spread via interactions with axonal pathways. We found that Schwann cells and hippocampal neurons were refractory to pseudorabies virus (PRV) infection transmitted from axons, suggesting a bottleneck to virus transmission. This work shows that $3 \mathrm{D}$ printing provides a valuable platform for the development of a customizable nervous system on a chip. Ultimately, 3D printed multiscale device architectures that reconstitute neural-tissue interfaces critical to organ function may expand the capabilities of cell culture models and provide viable alternatives to animal studies for fundamental research, drug screening, and toxicology applications. 


\section{Materials and methods}

\section{Harvesting and preparation of superior cervical ganglia and hippocampal neurons}

Primary embryonic sensory neurons from the superior cervical ganglia (SCG) and hippocampal neurons were obtained from Sprague-Dawley rats (embryonic day 15.5-16.5, Hilltop Labs Incorporated, Pennsylvania, United States). For each study, the animal was euthanized and handled in strict accordance with good animal practice as defined by the relevant national and local animal welfare bodies, and approved by the Princeton University Institutional Animal Care and Use Committee (IACUC). SCGs were first harvested and then dissociated. For the isolation of hippocampal neurons, hippocampal tissue was harvested from the same embryos used to obtain SCG neurons. A detailed procedure can be found elsewhere. ${ }^{38,39}$

\section{D printing of the nervous system on a chip}

Printer path information for the substrate microchannels and tri-chambers was constructed using vendor-provided software (Smart Robot, Fisnar) and computer-aided design (CAD) software (PTC Creo Parametric), respectively. CAD models were first converted to printer path information (G-code) using a cross-platform slicing program (KISSlicer), and subsequently converted to robot-specific language and transmitted to the robot using a custom LabVIEW program. 3D printing was done using a custom system consisting of a three-axis gantry industrial robot (F5200N, Fisnar, Wayne, NJ) with an external I/O card, a digital high precision dispenser (Ultimus V, Nordson EFD, Westlake, $\mathrm{OH}$ ), and a fixed stage (ThorLabs). Silicone (RTV, Loctite), polycaprolactone (Sigma), and cell suspensions were printed using extruder tips which ranged from 27 to 32 gauge (GA). Stage levelling within 1 $\mu \mathrm{m}$ was achieved using a digital CMOS laser sensor (Keyence, Itasca, IL). Two types of 35 $\mathrm{mm}$ dishes were used, plastic dishes (CELLTREAT) and dishes which contained glass inserts (MatTek Corporation). Printing was conducted directly on poly-L-ornithine and laminin-coated petri dishes.

\section{Functionalization and culturing of the 3DNSC}

Following 3D printing, the three individual chambers were functionalized with hippocampal neurons, SCG neurons, Schwann cells (S16, CRL-2941, ATCC), and epithelial cells (porcine kidney 15, PK-15 cells) according to the following procedures. Neurons were the first cells added to establish a robust axonal network prior to adding the Schwann and epithelial cells. Two chip designs were examined: 1) peripheral nervous system (PNS) chip models, and 2) central nervous system (CNS) chip models. SCG neurons were added to chamber 1 for PNS chips and chamber 2 for CNS chips. Hippocampal neurons were added to chamber 1 for CNS chips (PNS chips contained no hippocampal neurons). Neuronal cultures were maintained at $37{ }^{\circ} \mathrm{C}$ and $5 \% \mathrm{CO}_{2}$ and by replacing half of the medium every 5-7 days with fresh growth medium. After the axonal network established by the primary neurons penetrated into each of the individual chambers, which took 10-14 days, Schwann cells and epithelial cells were added to the other compartments, which lacked neurons but contained robust axonal networks. 


\section{Fluorescence microscopy}

Following cultivation of the mature chips, axons were stained for tau marker using anti-tau (monoclonal, mouse, Life Technologies), and epithelial cells were stained for cytokeratin marker using anti-cytokeratin (pan) (1:1000 in diluted blocking solution, polyclonal, rabbit, Life Technologies) and corresponding secondary antibodies (Alexa Flour 488 anti-mouse and Alexa Flour 568 anti-rabbit, Life Technologies). Imaging was carried out using a fluorescence microscope (Nikon Eclipse Ti, inverted epifluorescence microscope) as previously described. ${ }^{40}$ Axon-associated Schwann cells were imaged via a three-colour coinfection system that utilized a mixture of three separate viruses each containing a different fluorophore expression cassette (mCeruelean, EYFP, or mCherry), ${ }^{35}$ which provided enhanced resolution of axon-associated cell bodies.

\section{Design and propagation of viral strains}

All viral infection studies were conducted using pseudorabies virus (PRV). Epithelial cells were used to propagate and titre all PRV viral strains. PRV strains used in this study were previously constructed in our lab. ${ }^{35}$ Strains for two types of infections were examined: threecolour infections and two-colour infections. For three-colour infections, the viruses used were constructed from three plasmids containing a CMV-modified immediate-early promoter, which drives the expression of a fluorescent protein fused to a tandem triplet repeat of a nuclear localization signal (NLS). A detailed explanation of the design, construction and validation is provided elsewhere. ${ }^{41}$ Briefly, a EGFP-Us9 fusion gene was cloned into the $\mathrm{gG}$ locus of the PRV genome. This virus strain (designated PRV340) was then co-infected at a multiplicity of infection (MOI) of 10 PFUs (plaque forming units) per cell with a mRFP-VP26 Us9-null strain (PRV325) previously generated in our lab. ${ }^{42} \mathrm{We}$ then isolated and purified individual fluorescent plaques expressing both GFP and mRFP via three rounds of consecutive plaque purification, resulting in the dual labelled virus designated PRV 341.

\section{Quantification of viral transport}

Mature SCG neurons were infected at a MOI of 10 PFUs per cell with the dual colour virus PRV341 (mRFP-VP26 and EGFP-Us9) and incubated for 8-12 hr. Live imaging movies of the two-colour virus infections were acquired using near total internal reflectance fluorescence (TIRF) microscopy (also referred to as oblique angle microscopy) as previously described. ${ }^{43}$ Briefly, virus particles were imaged on a Nikon Ti-E microscope in the Princeton University Molecular Biology Confocal Microscopy Facility. This microscope was equipped with $488 \mathrm{~nm}$ and $561 \mathrm{~nm}$ excitation lasers (Agilent), an Apo TIRF 100X/1.49 NA oil immersion objective (Nikon), an Andor iXon Ultra EMCCD camera, a $37{ }^{\circ} \mathrm{C}$ heated stage, and Nikon NIS Elements software. Fluorescence emission bands were as follows: EGFP, $\sim 525 / 50 \mathrm{~nm}$ emission; mRFP, $\sim 605 / 50 \mathrm{~nm}$ emission. The velocity of individual virus particles was calculated as the distance travelled per elapsed time.

\section{Quantification of viral genome expression}

Mature CNS chips were infected in chamber 2, which contained SCG neurons with an inoculum containing an equal mixture of each of the three viral strains containing a 
fluorescent cassette resulting in approximately $1 \times 10^{6}$ PFUs of a three-colour inoculum. Following $24 \mathrm{hr}$ of incubation, directly infected cells in chamber 2 as well as hippocampal neurons and Schwann cells in chambers 1 and 3, respectively, were imaged to estimate the average number of viral genomes expressed per cell following infection of SCG neurons with PRV at a high MOI as previously described. ${ }^{44}$ Imaging was performed on a Nikon TiEclipse inverted microscope equipped with separate fast-switching excitation and emission filter wheels (Prior Scientific) using a Plan Fluor 20× Ph objective (Nikon). For each experimental condition, at least two experiments were performed with three replicates.

\section{Results and discussion}

\section{Design principle of the 3DNSC}

The nervous system is composed of a complex network of neurons, neurites, glia, and ECM. ${ }^{45}$ These components are organized into the CNS, consisting primarily of CNS neurons, astrocytes, and other supporting cells; and the PNS, consisting primarily of PNS neurons, axon-associated Schwann cells, and specialized axon termini that innervate peripheral tissues. ${ }^{45}$ As shown in Figure 1a, the inspiration for the 3DNSC is to provide a customizable, biomimetic in vitro model of the nervous system, with a one-to-one matching of the nervous system components within 3D printed platform chambers. Fabrication of the 3DNSC consisted of three steps, as outlined in Figure 1b:1) 3D printing of microchannels on a substrate to provide axonal guidance, 2) 3D printing of a substrate-chamber sealant layer to prevent fluid exchange among individual chambers, and 3) 3D printing of a top trichamber to provide isolation and organization of different cell types (e.g. neurons or glial cells). Following fabrication, the individual chambers were then functionalized with the desired cell types according to the mapping in Figure 1a to achieve a mature 3DNSC (see Figure 1c). One major advantage of this approach is the ability to readily establish separate fluid environments for neuronal axons and the soma from which they emanate. Thus, a perturbation to the nervous system - for example a viral inoculum - can be applied either to the compartment with the neuronal cell bodies, or to the compartment containing distal axons.

\section{Fabrication of the 3DNSC}

We initiated the manufacturing process by developing 3D computer models of both the substrate microchannels and the top tri-chamber, as shown schematically in Figures $1 \mathrm{~b}$ and 1c. As shown in Figure 1d, the $350 \mu \mathrm{m}$ wide 3D printed microchannels were constructed within a circular pattern (15 $\mathrm{mm}$ diameter) to optimize the percentage of cells on the chip which interact with axonal pathways. The width and inter-distance of the microchannels can be precisely tuned by controlling the dispensing time during the 3D printing process. As shown in Figure 1e, the top tri-chamber was $18 \mathrm{~mm}$ in diameter and $10 \mathrm{~mm}$ high, and contained two inner walls, which resulted in three adjacent $6 \mathrm{~mm}$ wide chambers. Computer models of the 3DNSC microchannels and tri-chamber components are provided in Figure S1 of the Supporting Information. The model for the sealant layer was identical to the top trichamber model, but the height was equal to that of a single layer $(\sim 150 \mu \mathrm{m})$. Both silicone and polycaprolactone were selected as the materials for direct microchannel printing onto protein-coated plastic dishes (printing time $=10 \mathrm{~min}$; see Movie S1). Following 
microchannel printing, the top tri-chamber and sealant layers were subsequently printed (printing time $=50 \mathrm{~min}$; see Movie S2). Silicone and grease served as the 3D printed materials for the tri-chamber and sealant layer, respectively. Thus, each 3DNSC required ca. one hour in total to fabricate. This throughput may be improved in the future via the use of multiplexed extrusion approaches, or by increasing the linear speed of the extruder, which was in the range of $0.1-1 \mathrm{~mm} \mathrm{~s}^{-1}$. Assembly of the 3D printed substrate and top chamber components resulted in a complete 3DNSC. A distinguishing feature of the 3D printed in vitro systems relative to conventional compartmented chamber and microfluidic systems is the rapid prototyping capability, which we highlight in Figures S2-S5.

\section{Functionalization and biomimetic maturation of the 3DNSC}

We next functionalized the individual chambers of the chip with appropriate cell types. As a proof of concept, we also examined the functionalization of the chambers via direct 3D printing of cells (see Figure S1). As shown in Figure 1a, the nervous system may be modelled as four primary interacting components which are connected by axons: 1) CNS neurons, 2) PNS neurons, 3) Schwann cells, and 4) epithelial cells. Thus, we examined two 3DNSC models with the tri-chamber (1-2-3) design: a peripheral 3DNSC consisting of PNS neurons-Schwann cells-epithelial cells, and a central 3DNSC consisting of CNS neuronsPNS neurons-Schwann cells. We first developed a peripheral 3DNSC. As shown in Figure 2a, a peripheral 3DNSC was constructed which contained SCG neurons in chamber 1 (1), Schwann cells in chamber 2 (2), and PK-15 cells in chamber 3 (3). Figures 2b-g show the alignment of the axons and the organization of cell types within the printed microchannels. Associated phase contrast micrographs can be found in Figure S6.

Several important conclusions can be drawn from Figures $2 b-g$, which reveal effective biomimicry of the nervous system: 1) spatial separation of different cell types is achieved over micrometre to centimetre scales, 2) Schwann cells spontaneously associated with axons (see Figure S7), and 3) the axonal network penetrates into all chambers, providing the opportunity for axon-to-cell connections between the organized cells. Additional analysis of Schwann cell-axon association is provided in Figure S7. Given the complexity of the nervous system, there are many native physical (e.g. topographical, mechanical, and structural), biochemical, and cellular features which may ultimately be mimicked. Our proof-of-concept approach for demonstrating the application of 3D printing for in vitro model manufacturing involves the CNS-PNS junction. Therefore, focus here is placed on biomimicry of the cellular compartmentalization and topography, which establishes highly aligned axonal networks which contain associated Schwann cells.

\section{In vitro model for physiologically relevant viral infection assays}

Having shown that 3DNSCs exhibit biomimetic structures, we next focused on applying the chip to physiologically relevant organ-level viral infection studies. An understanding of viral infection and transport in the nervous system is critical to developing future treatments for various neurological diseases and disorders. ${ }^{46,47}$ Therefore, we created 3DNSCs containing additional CNS components, i.e., central 3DNSCs. 
The central 3DNSC design is shown in Figure 3a and consists of (1) CNS neurons (hippocampal neurons) in chamber 1, (2) PNS neurons (SCG neurons) in chamber 2, and (3) Schwann cells in chamber 3. As shown schematically in Figure 3a, the viral assay involves infecting only chamber 2 - which contains the PNS neurons (SCGs) - with pseudorabies virus (PRV). We then monitored the axon-to-cell spread of viral particles to both hippocampal neurons (chamber 1) and Schwann cells (chamber 3).

As shown in Figure 3b, we used a two-colour infection assay combined with oblique angle fluorescence microscopy to show that: 1) SCG neurons were infected by PRV, and 2) PRV was replicated by the SCG neurons, as indicated by the observation of immature (red capsid) and mature virus particles (yellow puncta, a result of the red capsid tag co-localizing with the green envelope protein, suggesting a fully assembled particle) in the nucleus and axons. Rapid prototyping of chips on glass substrates also enabled calculation of the virus axonal transport rate as $2.0 \mu \mathrm{m} \mathrm{s}^{-1}$ via high-resolution imaging studies (Figure S5). The results shown in Figure 3b suggest that SCG neurons are infected by PRV, and viral particles are transported within the axonal network of chamber 2 (the point of infection).

We next examined the spread of infectious particles to the other regions of the 3DNSC. We used a three-colour ("Brainbow") infection assay, which functions on the principle that if SCGs are co-infected with multiple viruses expressing primary colours and spread many infectious units to subsequent cells (e.g. hippocampal and Schwann cells), then one would expect those cells to also express multiple colours. Given the high number of progeny viruses produced from a single infected cell, we would expect multiple infection events in each cell connected to the cell replicating the virus. As shown by the representative images in Figures 3c-f, direct infection of PNS neurons (SCGs) under high MOI indeed led to replication and spread of infection to the CNS neurons and Schwann cells. However, as indicated by the fluorescence signal, CNS neurons and Schwann cells exhibited only single primary colours, and not a multicolour profile, suggesting that there is a bottleneck to virus transmission from PNS axons to these cell types. This bottleneck to viral spread and infection has been previously observed in other cell types and suggests that there is a viral or cellular factor limiting infection of a single cell by multiple virons. ${ }^{35,44,48,49}$

Given that the recombinant viruses are engineered to express primary colours when a single viral infection occurs, if more than one viral particle infects a cell, the colour profile will be altered, enabling one to estimate the number of virus genomes per cell via the following relation: ${ }^{35,44}$

$$
\lambda=-3 \ln \left(1-\frac{r_{1}+2 r_{2}+3 r_{3}}{3 n}\right)
$$

where $\lambda$ is the number of genomes expressed in each cell, $r_{1}$ is the number of one-colour cells, $r_{2}$ is the number of two-colour cells, $r_{3}$ is the number of three-colour cells, and $n$ is the number of cells analysed. As shown in Figure 3c, hippocampal neurons and Schwann cells showed similar restriction of viral uptake as indicated by 1.6 and 1.4 genomes per cell, respectively. This result reveals two new observations enabled by the 3DNSCs which are 
important for understanding infection mechanisms and barriers in the nervous system: 1) hippocampal neuron and Schwann cell types appear to be refractory to infection, and 2) Schwann cells appear to participate in infection response through axonal interaction. Importantly, the collective results in Figure 3 demonstrate that 3DNSCs provide a flexible physiological platform for virology, molecular biology, and neuroscience assays which require the interrogation of system-level responses to localized perturbation (e.g. drugs, pathogens, electrical/mechanical energy, etc.).

\section{Conclusions}

In conclusion, 3D printing was used to create a nervous system on a chip by providing automated assembly of chambers and customized design of the microchannels and the chamber geometry. Viral assays were applied to demonstrate biological connectivity among the individual cellular components (e.g. neurons and glia), and to illustrate the flexibility and utility of the technology. Schwann cells and hippocampal neurons were found to be refractory to axon-to-cell infection of pseudorabies virus, suggesting a bottleneck to viral transmission. The successful integration of multiple neural components suggests that additive manufacturing may be used as an effective fabrication approach for the development of customizable organ-on-a-chip technologies. Future work will focus on: 1) direct patterning of neurons and glia, 2) extending this work to $3 \mathrm{D}$ microchannel networks, ${ }^{50}$ 3 ) imaging of synapses and other close cell-cell contacts, 4) introducing CNS glial components, 5) examination of the neural electrical activity on physiological function, and 6) customization of CNS chips focused specifically on brain models.

\section{Supplementary Material}

Refer to Web version on PubMed Central for supplementary material.

\section{Acknowledgments}

We thank Dr. Paul Shao, Princeton Institute for the Science and Technology of Materials, and Department of Molecular Biology, for consultation and assistance with electron microscopy. B.N.J. and K.Z.L. contributed equally to this work. I.B.H. acknowledges the support of the American Cancer Society fellowship (PF-13-050-01-MPC). L.W.E. acknowledges the support of this work by the National Institutes of Health (NIH Grant No. R01NS033506 and Grant No. R01NS060699). M.C.M. acknowledges the support of the Defence Advanced Research Project Agency (DARPA Grant No. D12AP00245), and the Grand Challenges Program at Princeton University.

\section{References}

1. Huh D, Matthews BD, Mammoto A, Montoya-Zavala M, Hsin HY, Ingber DE. Science. 2010; 328:1662-1668. [PubMed: 20576885]

2. Beebe DJ, Ingber DE, den Toonder J. Lab Chip. 2013; 13:3447-3448. [PubMed: 23918086]

3. Huh D, Leslie DC, Matthews BD, Fraser JP, Jurek S, Hamilton GA, Thorneloe KS, McAlexander MA, Ingber DE. Sci Transl Med. 2012; 4:159ra147.

4. van der Meer AD, van den Berg A. Integr Biol. 2012; 4:461-470.

5. Yum K, Hong SG, Healy KE, Lee LP. Biotechnol J. 2014; 9:16-27. [PubMed: 24357624]

6. Sung JH, Esch MB, Prot JM, Long CJ, Smith A, Hickman JJ, Shuler ML. Lab Chip. 2013; 13:12011212. [PubMed: 23388858] 
7. Shintu L, Baudoin R, Navratil V, Prot JM, Pontoizeau C, Defernez M, Blaise BJ, Domange C, Péry AR, Toulhoat P, Legallais C, Brochot C, Leclerc E, Dumas ME. Anal Chem. 2012; 84:1840-1848. [PubMed: 22242722]

8. Booth R, Kim H. Lab Chip. 2012; 12:1784-1792. [PubMed: 22422217]

9. Calvert P. Chem Mater. 2001; 13:3299-3305.

10. Mironov V, Boland T, Trusk T, Forgacs G, Markwald RR. Trends Biotechnol. 2003; 21:157-161. [PubMed: 12679063]

11. Curodeau A, Sachs E, Caldarise S. J Biomed Mater Res. 2000; 53:525-535. [PubMed: 10984701]

12. Khalil S, Nam J, Sun W. Rapid Prototyp J. 2005; 11:9-17.

13. Sachs E, Cima M, Cornie J, Brancazio D, Bredt J, Curodeau A, Fan T, Khanuja S, Lauder A, Lee J, Michaels S. CIRP Ann Manuf Technol. 1993; 42:257-260.

14. Ahn BY, Duoss EB, Motala MJ, Guo X, Park SI, Xiong Y, Yoon J, Nuzzo RG, Rogers JA, Lewis JA. Science. 2009; 323:1590-1593. [PubMed: 19213878]

15. Frutiger A, Muth JT, Vogt DM, Mengüç Y, Campo A, Valentine AD, Walsh CJ, Lewis JA. Adv Mater. 2015; 27:2440-2446. [PubMed: 25754237]

16. Kong YL, Tamargo IA, Kim H, Johnson BN, Gupta MK, Koh TW, Chin HA, Steingart DA, Rand BP, McAlpine MC. Nano Lett. 2014; 14:7017-7023. [PubMed: 25360485]

17. Chang JW, Park SA, Park JK, Choi JW, Kim YS, Shin YS, Kim CH. Artif Organs. 2014; 38:E95E105. [PubMed: 24750044]

18. Duan B, Hockaday LA, Kang KH, Butcher JT. J Biomed Mater Res, Part A. 2013; 101A:12551264.

19. Hockaday LA, Kang KH, Colangelo NW, Cheung PY, Duan B, Malone E, Wu J, Girardi LN, Bonassar LJ, Lipson H, Chu CC, Butcher JT. Biofabrication. 2012; 4:035005. [PubMed: 22914604]

20. Herbert N, Simpson D, Spence W, Ion W. J Rehabil Res Dev. 2005; 42:141-146. [PubMed: 15944878]

21. Mannoor MS, Jiang Z, James T, Kong YL, Malatesta KA, Soboyejo WO, Verma N, Gracias DH, McAlpine MC. Nano Lett. 2013; 13:2634-2639. [PubMed: 23635097]

22. Ahn SH, Lee KT, Kim HJ, Wu R, Kim JS, Song SH. Intl J Prec Eng Manuf. 2012; 13:631-634.

23. Roth EA, Xu T, Das M, Gregory C, Hickman JJ, Boland T. Biomaterials. 2004; 25:3707-3715. [PubMed: 15020146]

24. Cohen DL, Malone E, Lipson H, Bonassar LJ. Tissue Eng. 2006; 12:1325-1335. [PubMed: 16771645]

25. Pfister A, Landers R, Laib A, Hübner U, Schmelzeisen R, Mülhaupt R. J Polym Sci, Part A: Polym Chem. 2004; 42:624-638.

26. Murphy SV, Atala A. Nat Biotechnol. 2014; 32:773-785. [PubMed: 25093879]

27. Mironov V, Reis N, Derby B. Tissue Eng. 2006; 12:631-634. [PubMed: 16674278]

28. Krejcova L, Nejdl L, Rodrigo MAM, Zurek M, Matousek M, Hynek D, Zitka O, Kopel P, Adam V, Kizek R. Biosens Bioelectron. 2014; 54:421-427. [PubMed: 24296063]

29. Gonzalez-Macia L, Morrin A, Smyth MR, Killard AJ. Analyst. 2010; 135:845-867. [PubMed: 20419231]

30. Rossiter J, Walters P, Stoimenov B. Proc SPIE EAPAD. 2009; 7287:72870H.

31. Matsusaki M, Sakaue K, Kadowaki K, Akashi M. Adv Healthc Mater. 2013; 2:534-539. [PubMed: 23184899]

32. Lee JB, Sung JH. Biotechnol J. 2013; 8:1258-1266. [PubMed: 24038956]

33. Wagner I, Materne E-M, Brincker S, Su, Fradrich C, Busek M, Sonntag F, Sakharov DA, Trushkin EV, Tonevitsky AG, Lauster R, Marx U. Lab Chip. 2013; 13:3538-3547. [PubMed: 23648632]

34. Schimek K, Busek M, Brincker S, Groth B, Hoffmann S, Lauster R, Lindner G, Lorenz A, Menzel U, Sonntag F, Walles H, Marx U, Horland R. Lab Chip. 2013; 13:3588-3598. [PubMed: 23743770]

35. Taylor MP, Kobiler O, Enquist LW. Proc Natl Acad Sci USA. 2012; 109:17046-17051. [PubMed: 23027939] 
36. Sunja K, Jaewon P, Arum H, Jianrong L. Neural Regen Res. 2014; 9:1703-1705.

37. Johnson BN, Lancaster KZ, Zhen G, He J, Gupta MK, Kong YL, Engel EA, Krick KD, Ju A, Meng F, Enquist LW, Jia X, McAlpine MC. Adv Funct Mater. 2015; 25:6205-6217. [PubMed: 26924958]

38. Curanović, D., Ch'ng, TH., Szpara, M., Enquist, L. Current Protocols in Cell Biology. Vol. 43. John Wiley \& Sons, Inc; 2009. p. 26.24.21-26.24.23.

39. Ch'ng TH, Enquist LW. J Virol. 2005; 79:10875-10889. [PubMed: 16103140]

40. Taylor M, Kratchmarov R, Enquist L. J Vis Exp. 2013; 78doi: 10.3791/50723

41. Taylor MP, Kramer T, Lyman MG, Kratchmarov R, Enquist LW. mBio. 2012; 3:e00063-00012. [PubMed: 22448044]

42. Lyman MG, Feierbach B, Curanovic D, Bisher M, Enquist LW. J Virol. 2007; 81:11363-11371. [PubMed: 17686845]

43. Hogue IB, Bosse JB, Hu JR, Thiberge SY, Enquist LW. PLoS Pathog. 2014; 10:e1004535. [PubMed: 25474634]

44. Kobiler O, Lipman Y, Therkelsen K, Daubechies I, Enquist LW. Nat Commun. 2010; 1:146. [PubMed: 21266996]

45. Brodal, A. Neurological anatomy in relation to clinical medicine. Oxford University Press; New York, USA: 1981.

46. Pomeranz LE, Reynolds AE, Hengartner CJ. Microbiol Mol Biol Rev. 2005; 69:462-500. [PubMed: 16148307]

47. Crawford JR. Curr Neurol Neurosci Rep. 2010; 10:147-154. [PubMed: 20425240]

48. Pfeiffer JK, Kirkegaard K. Proc Natl Acad Sci USA. 2006; 103:5520-5525. [PubMed: 16567621]

49. Lancaster KZ, Pfeiffer JK. PLoS Pathog. 2010; 6:e1000791. [PubMed: 20221252]

50. Pautot S, Wyart C, Isacoff EY. Nat Meth. 2008; 5:735-740. 

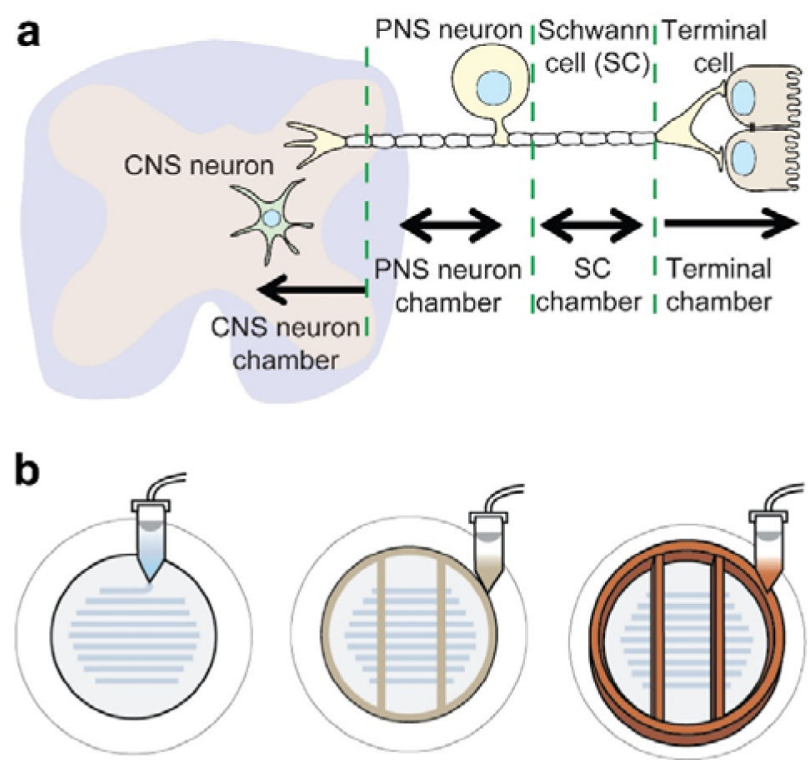

I) Channel Printing II) Seal Printing III) Chamber Printing

C
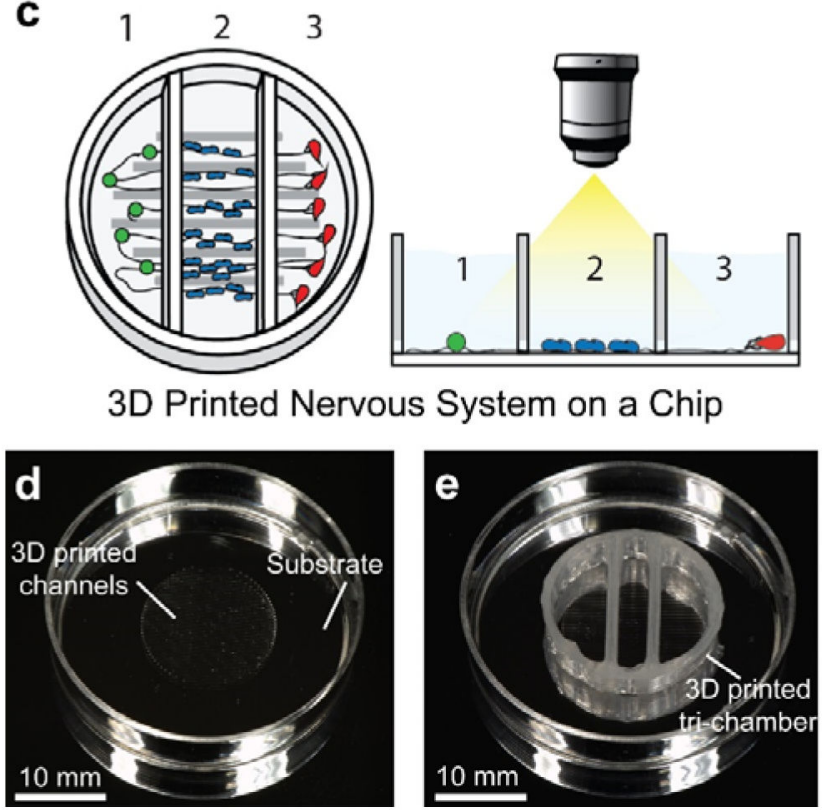

Fig. 1.

a) Schematic of the nervous system, modelled as four primary components: CNS neurons, PNS neurons, axon-associated Schwann cells, and epithelial cells. b) 3D printing of the model nervous system on a chip, consisting of (I) parallel microchannels, (II) a sealant layer, and (III) a top tri-chamber. c) Schematic of a representative 3DNSC for peripheral nervous system applications, showing (1) PNS neurons in chamber 1, (2) Schwann cells in chamber 2 , and (3) terminal cell junctions in chamber 3 . The Schwann cells and the terminal cells interact with the neurons and each other solely via the axonal network. d) Circular pattern of 3D printed silicone microchannels for axonal guidance in the centre of a plastic $35 \mathrm{~mm}$ dish. 
e) A 3DNSC showing perpendicular assembly of microchannel and tri-chamber components. 
a

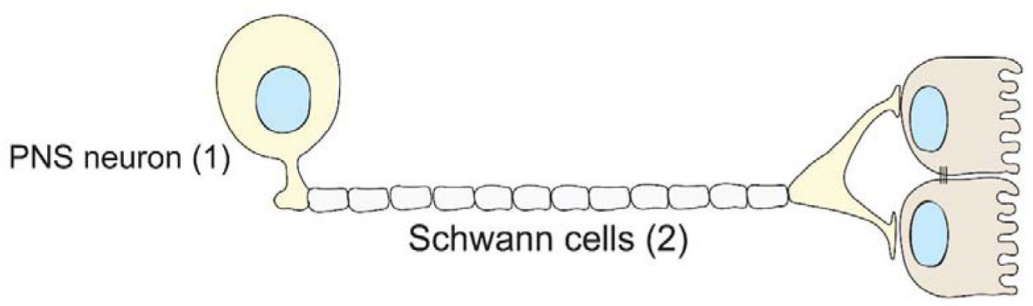

Cell junction (3)
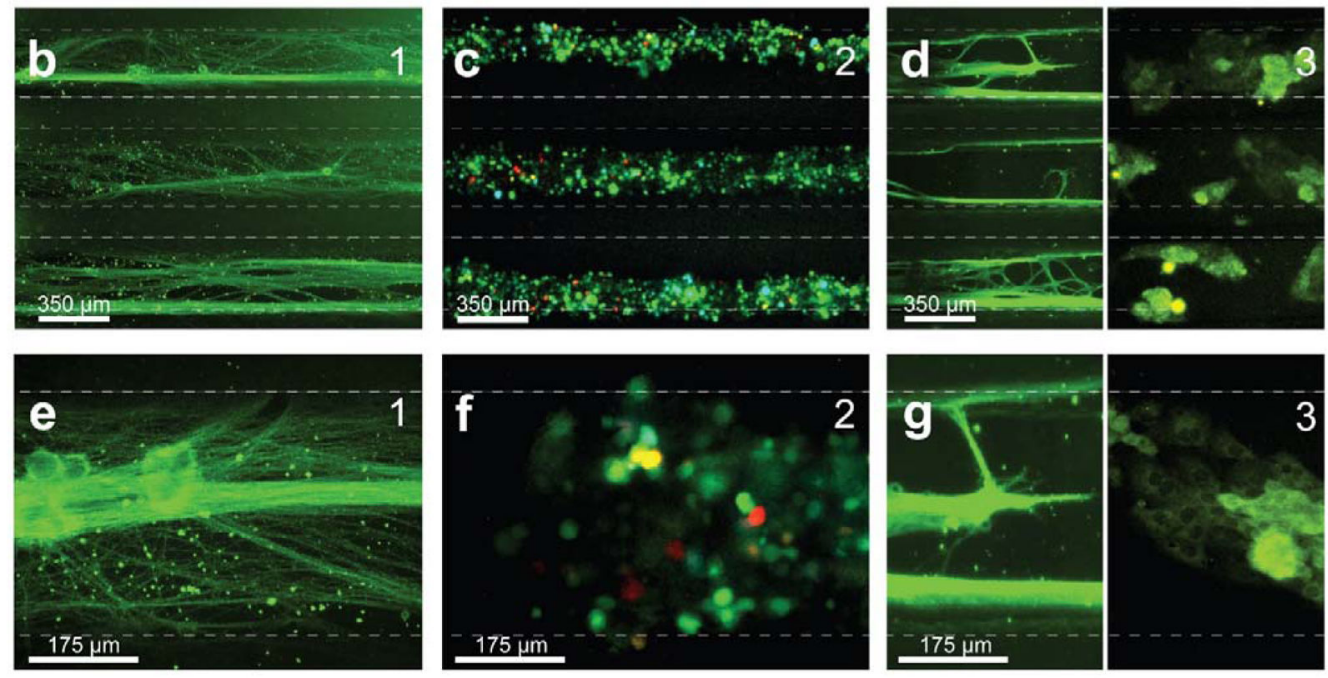

Fig. 2.

a) Schematic of a peripheral 3DNSC showing three primary components: (1) the neuronal component and source of axons containing SCG neurons in chamber 1, (2) the peripheral nerve component containing axons and Schwann cells in chamber 2, and (3) the terminal cell junction component containing axon termini and epithelial cells in chamber 3. b) Micrograph showing three parallel microchannels of SCG neurons and axons (green tau stained) in chamber 1. c) Micrograph showing three parallel channels of peripheral nerve fibres containing organized self-assembled networks of axon-associated Schwann cells (tricolour PRV Brainbow strain; see Materials and methods section) in chamber 2. d) Micrograph showing three parallel channels of axon termini (green tau stained) and epithelial cells (green cytokeratin stained) in chamber 3. e) Single channel image of neurons from (b). f) Single channel image of Schwann cells from (c). g) Single channel image of axon termini and epithelial cells from (d). Phase contrast images are provided in Figure S6. 


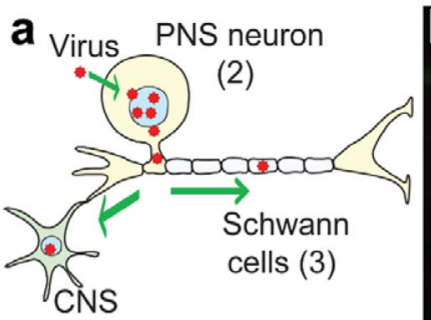

neuron (1)

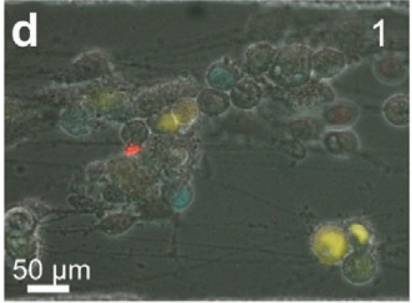

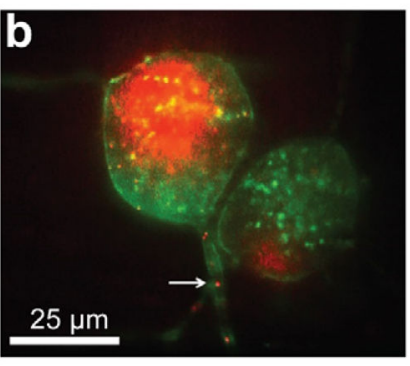
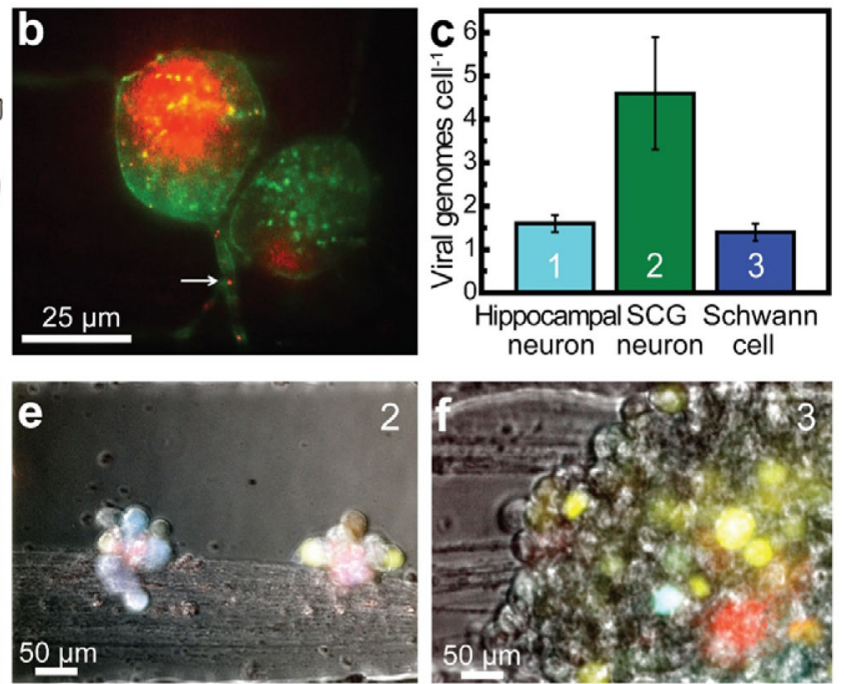

Fig. 3.

a) Schematic of a central 3DNSC showing three primary components: (1) the CNS neuronal component containing hippocampal neurons in chamber 1, (2) the PNS neuronal component containing SCG neurons and sources of axons in chamber 2, and (3) the peripheral nerve component containing axons and Schwann cells in chamber 3. Arrows schematically depict the model viral infection assay, which involves infection of neurons in the PNS, and spread of the infection to the CNS neurons and Schwann cells. b) Total internal reflectance fluorescence (TIRF) micrograph of infected PNS neurons in chamber 2, with PRV twocolour viral strains showing replication and axonal transport of viral particles (arrow highlights a single virus particle). c) Estimated level of viral genome expression in infected cells found in chambers 1 and 3, originating from high MOI of PNS neurons in chamber 2. d) Micrograph of infected hippocampal neurons (1; PRV three-colour strains). e) Micrograph of infected SCG neurons (2; PRV three-colour strains). f) Micrograph of infected Schwann cells (3; PRV three-colour strains). 